\title{
THE
}

4-15-1996

\section{Impact of Criticality and Phase Separation on the Spin Dynamics of the One-Dimensional $\mathrm{t}-\mathrm{J}$ Model}

\author{
Shu Zhang \\ University of Rhode Island \\ Gerhard Müller \\ University of Rhode Island, gmuller@uri.edu \\ Joachim Stolze
}

Follow this and additional works at: https://digitalcommons.uri.edu/phys_facpubs

Terms of Use

All rights reserved under copyright.

\section{Citation/Publisher Attribution}

Shu Zhang, Gerhard Müller and Joachim Stolze. Impact of criticality and phase separation on the spin dynamics of the one-dimensional t-J model. J. Appl. Phys. 79 (1996), 5227-5229.

Available at http://dx.doi.org/10.1063/1.361349.

This Article is brought to you for free and open access by the Physics at DigitalCommons@URI. It has been accepted for inclusion in Physics Faculty Publications by an authorized administrator of DigitalCommons@URI. For more information, please contact digitalcommons-group@uri.edu. 


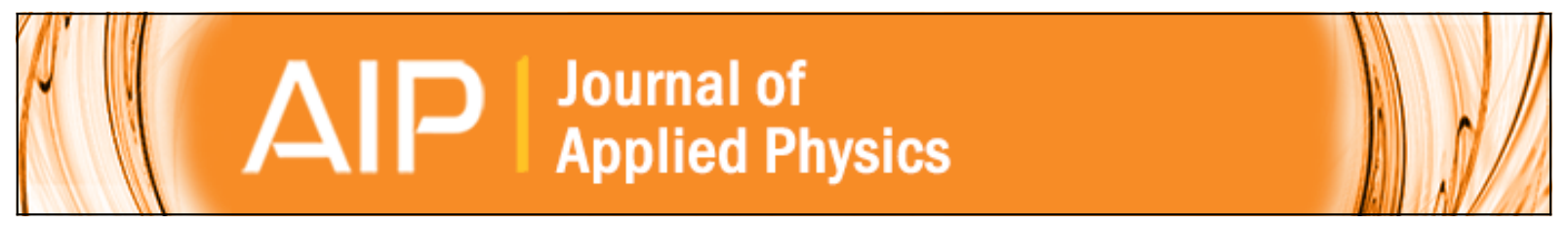

Impact of criticality and phase separation on the spin dynamics of the onedimensional $\mathbf{t}-\mathbf{J}$ model

Shu Zhang, Gerhard Müller, and Joachim Stolze

Citation: Journal of Applied Physics 79, 5227 (1996); doi: 10.1063/1.361349

View online: http://dx.doi.org/10.1063/1.361349

View Table of Contents: http://scitation.aip.org/content/aip/journal/jap/79/8?ver=pdfcov

Published by the AIP Publishing

\section{Articles you may be interested in}

A one-dimensional phase transition constitutive model

AIP Conf. Proc. 505, 347 (2000); 10.1063/1.1303489

Spin coating: Onedimensional model

J. Appl. Phys. 66, 5185 (1989); 10.1063/1.343754

Observation of the dynamic behavior of the antiferromagneticferromagnetic phase transition in the one dimensional spin $1 / 2$ antiferromagnet $\alpha \mathrm{CuNSal}$

J. Appl. Phys. 50, 1859 (1979); 10.1063/1.327145

OneDimensional Ising Model with General Spin

J. Math. Phys. 8, 124 (1967); 10.1063/1.1705089

OneDimensional Phase Transition in the Spherical Model of a Gas

Phys. Fluids 6, 599 (1963); 10.1063/1.1706787

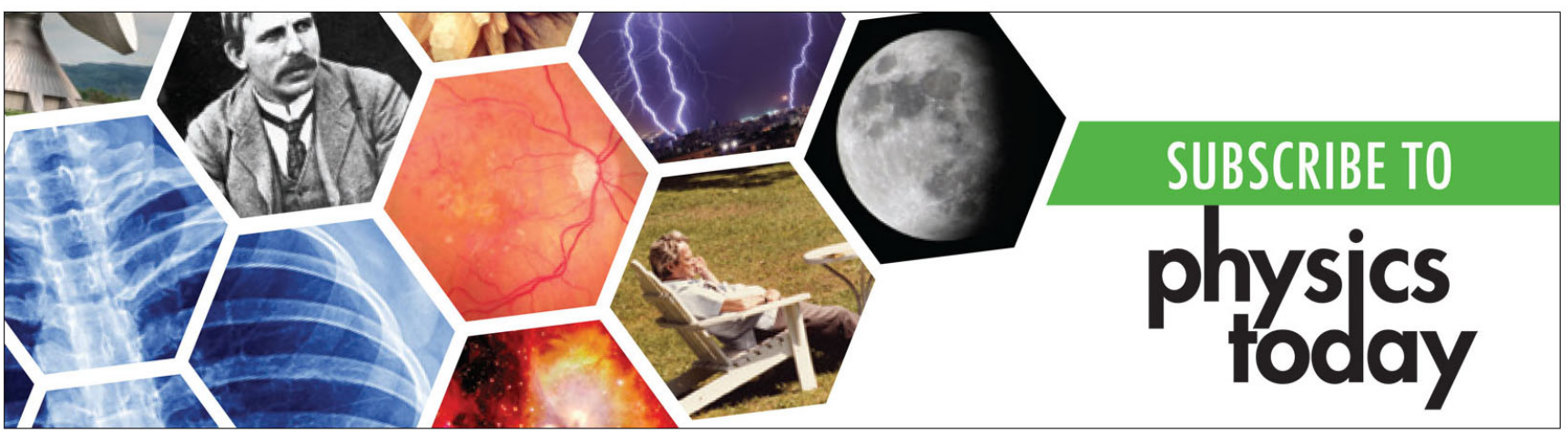




\title{
Impact of criticality and phase separation on the spin dynamics of the one-dimensional $t-J$ model
}

\author{
Shu Zhang and Gerhard Müller \\ Department of Physics, The University of Rhode Island, Kingston, Rhode Island 02881-0817
}

Joachim Stolze ${ }^{\text {a) }}$

Physikalisches Institut, Universität Bayreuth, 95440 Bayreuth, Germany

The recursion method is used to determine the $T=0$ spin dynamic structure factor $S_{z z}(q, \omega)$ in the Luttinger liquid state and in the phase-separated state of the one-dimensional $t-J$ model. As the exchange coupling increases from zero, the dispersions and line shapes of the dominant spin excitations are observed to undergo a major metamorphosis between the free-fermion limit and the onset of phase separation. The familiar two-spinon spectrum of the Heisenberg antiferromagnetic chain emerges gradually in the strongly phase-separated state. (C) 1996 American Institute of Physics. [S0021-8979(96)18608-0]

The $t-J$ model for strongly correlated electrons has been widely used as a prototypical system for the study of the interplay between charge and spin degrees of freedom. ${ }^{1}$ For a one-dimensional (1D) lattice of $N$ sites the Hamiltonian reads

$$
\begin{aligned}
H_{t-J}= & -t \sum_{\sigma=\uparrow, \downarrow} \sum_{l=1}^{N}\left\{\tilde{c}_{l, \sigma}^{\dagger} \tilde{c}_{l+1, \sigma}+\tilde{c}_{l+1, \sigma}^{\dagger} \tilde{c}_{l, \sigma}\right\} \\
& +J \sum_{l=1}^{N}\left\{\mathbf{S}_{l} \cdot \mathbf{S}_{l+1}-\frac{1}{4} n_{l} n_{l+1}\right\}
\end{aligned}
$$

with $\tilde{c}_{l, \sigma}=c_{l, \sigma}\left(1-n_{l,-\sigma}\right), n_{l}=n_{l, \uparrow}+n_{l, \downarrow}, n_{l, \sigma}=c_{l, \sigma}^{\dagger} c_{l, \sigma}$, $S_{l}^{z}=\left(n_{l, \uparrow}-n_{l, \downarrow}\right) / 2$, and $S_{l}^{+}=\tilde{c}_{l, \uparrow}^{\dagger} \tilde{c}_{l, \downarrow}$. Here we consider the quarter-filled-band case ( $N_{e}=N / 2$ electrons).

The hopping term amounts to an effectively repulsive force between electrons on nearest-neighbor sites, ${ }^{2}$ and the exchange term represents an attractive force if these two electrons have opposite spins. As the exchange interaction increases from zero, the ground state of $H_{t-J}$ undergoes a transition, at $J / t \simeq 3.2$, from a Luttinger liquid state to a phase-separated state. ${ }^{3-5}$ In the Luttinger liquid state, both the charge correlations (at $q=\pi$ ) and the spin correlations (at $q=\pi / 2)$ are critical. The transition, which is driven by the spin coupling, produces charge long-range order (at $q=0$ ) combined with a new type of spin criticality (at $q=\pi$ ).

The focus of this study is on the frequency-dependent spin fluctuations of $H_{t-J}$ as they manifest themselves in the $T=0$ spin dynamic structure factor

$$
S_{z z}(q, \omega)=\frac{1}{N} \sum_{l, m} e^{-i q m} \int_{-\infty}^{+\infty} d t e^{i \omega t}\left\langle S_{l}^{z}(t) S_{l+m}^{z}\right\rangle .
$$

For the calculation of this quantity, we employ the recursion method $^{6}$ in combination with a strong-coupling continuedfraction analysis. ${ }^{7,8}$ The recursion method in the present context is based on an orthogonal expansion of the wave function $\left|\Psi_{q}^{z}(t)\right\rangle=S_{q}^{z}(-t)|G\rangle$, where $S_{q}^{z}=N^{-1 / 2} \Sigma_{l} e^{i q l} S_{l}^{z}$ is the spin fluctuation operator, and $|G\rangle$ is the finite-size groundstate wave function of Eq. (1). After some intermediate steps,

${ }^{a)}$ Permanent address: Institut für Physik, Universitat Dortmund, 44221 Dortmund, Germany. the algorithm produces a sequence of continued-fraction coefficients $\Delta_{1}^{z}(q), \Delta_{2}^{z}(q), \ldots$ for the relaxation function,

$$
c_{0}^{z z}(q, z)=\frac{1}{z+\frac{\Delta_{1}^{z}(q)}{z+\frac{\Delta_{2}^{z}(q)}{z+\cdots}}},
$$

which is the Laplace transform of the symmetrized correlation function $\mathfrak{R}\left\langle S_{q}^{z}(t) S_{-q}^{z}\right\rangle /\left\langle S_{q}^{z} S_{-q}^{z}\right\rangle$. The $T=0$ spin dynamic structure factor (2) is then obtained from Eq. (3) via the relation

$$
S_{z z}(q, \omega)=4\left\langle S_{q}^{z} S_{-q}^{z}\right\rangle \Theta(\omega) \lim _{\epsilon \rightarrow 0} \Re\left[c_{0}^{z z}(q, \epsilon-i \omega)\right] .
$$

In a previous paper ${ }^{9}$ we have used the recursion method together with a weak-coupling continued-fraction analysis for a study of the charge dynamic structure factor in the Luttinger liquid phase of the $t-J$ model. The spin dynamics poses a far greater challenge. Very few explicit results seem to exist. ${ }^{10}$ One key spin dynamical property in the Luttinger liquid phase can be inferred from the asymptotic behavior of the static spin correlations as proposed in previous work: ${ }^{4,5}$

$$
\left\langle S_{l}^{z} S_{l+m}^{z}\right\rangle \sim B_{1} \frac{1}{m^{2}}+B_{2} \frac{\cos (\pi m / 2)}{m^{\eta_{\rho} / 4+1}},
$$

where $\eta_{\rho}$ is the exponent which also governs the algebraic decay of the $(q=\pi)$ oscillations, $\sim \cos (\pi m) / m^{\eta_{\rho}}$, in the static charge correlation function $\left\langle n_{l} n_{l+m}\right\rangle$. This exponent is known to assume the value $\eta_{\rho}=2$ in the free-fermion limit $\left(\mathrm{J} / t=0^{+}\right)$. It increases linearly, $\Delta \eta_{\rho} \simeq 0.40 \mathrm{~J} / t$, in the weakcoupling regime, ${ }^{3,9}$ assumes the value $\eta_{\rho} \simeq 3.4$ at $J / t=2$ (supersymmetric case), ${ }^{11}$ and diverges at the endpoint, $J / t \simeq 3.2$, of the Luttinger liquid state. ${ }^{3}$

The oscillatory term in Eq. (5) implies that the dynamically relevant excitation spectrum of $S_{z z}(q, \omega)$ is gapless at $q=\pi / 2$. The spectral-weight distribution of the spin dynamic structure factor at this critical wave number can then be predicted (under mild assumptions) to have a singularity of the form

$$
S_{z z}(\pi / 2, \omega) \sim \omega^{\eta_{\rho} / 4-1} .
$$

The infrared exponent starts out negative in the free-fermion limit, $\sim \omega^{-1 / 2}$, increases monotonically with increasing $J / t$, 


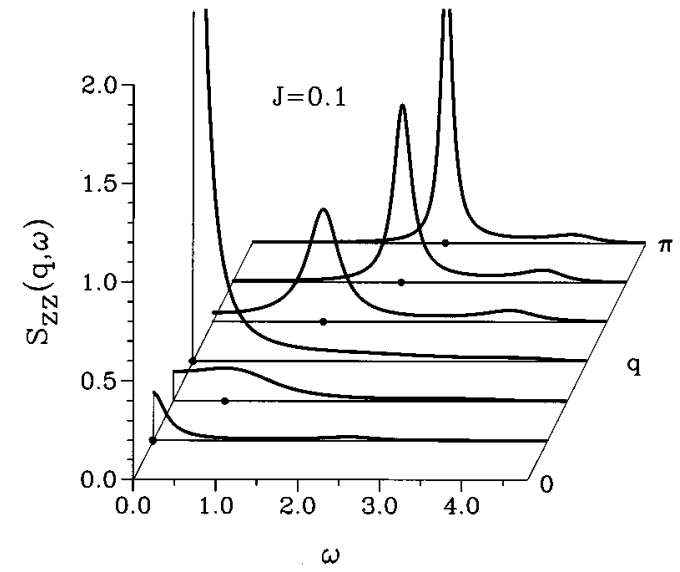

FIG. 1. Spin dynamic structure factor $S_{z z}(q, \omega)$ at $T=0$ for $q=2 \pi l / 12$, $l=0, \ldots, 6$ of the $1 \mathrm{D} t-J$ model with $t=1$ and $J=0.1$ in the Luttinger liquid phase near the free-fermion limit.

and then diverges at $J / t \simeq 3.2$, where phase separation sets in. A landmark change in $S_{z z}(\pi, \omega)$ is expected to occur at the point where the infrared exponent switches sign (from negative to positive). This happens for $\eta_{\rho}=4$, which corresponds to the coupling strength $J / t \simeq 2.3$.

Our results for $S_{z z}(q, \omega)$ indicate that the Luttinger liquid phase of the $t-J$ model can be divided into two regimes with distinct spin dynamical properties. As a representative result of the first regime $(0<J / t \leqslant 1)$, we show in Fig. 1 $S_{z z}(q, \omega)$ as a continuous function of $\omega$ and a discrete function of the $q$ values realized in a system of $N=12$ sites with coupling strength $J / t=0.1$.

All results presented here have been calculated via a strong-coupling continued-fraction reconstruction from the coefficients $\Delta_{1}, \ldots, \Delta_{6}$ and a Gaussian terminator. The $\Delta_{k}$ 's have been extracted via the recursion method from the ground-state wave function for $N=12$, which in turn has been computed via the conjugate-gradient method. The entire procedure was explained in Ref. 7.

Throughout the Brillouin zone except at small $q$ we observe a well-defined dynamically relevant spin mode with a $|\cos q|$-like dispersion as indicated by the full circles. Near the critical wave number, $q=\pi / 2$, the function $S_{z z}(q, \omega)$ may exhibit a power-law divergence of the form $\sim\left[\omega^{2}\right.$ $\left.-c^{2}(\pi / 2-q)^{2}\right]^{\left(\eta_{\rho} / 4-1\right) / 2}$, similar to what has been observed at the critical wave number in other Luttinger liquids. ${ }^{12}$ At long wavelengths the spectral weight in $S_{z z}(q, \omega)$ is concentrated at fairly low frequencies. Data for longer chains will be needed for a quantitative analysis of the spin dispersions at small $q$ in this regime.

As the exchange coupling increases toward $J / t \simeq 1$, the following changes in the spectral-weight distribution of $S_{z z}(q, \omega)$ can be identified:

(i) The amplitude of the $|\cos q|$-like dispersion grows with increasing $J / t$. The gradual upward shift of the peak position in $S_{z z}(q, \omega)$ is accompanied by a significant increase in linewidth. For the $q=\pi$ spin mode this is contrary to what one expects under the influence of an antiferromagnetic exchange interaction of increasing strength. That trend changes at stronger coupling as we shall see.

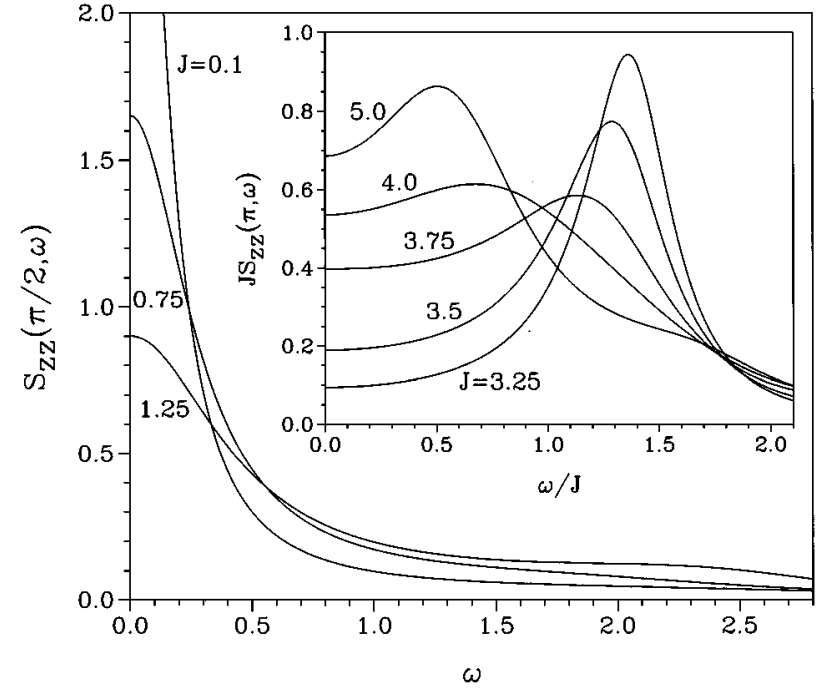

FIG. 2. Line shape at $q=\pi / 2$ of the spin dynamic structure factor $S_{z z}(q, \omega)$ at $T=0$ for various values of $J$ in the Luttinger liquid state of the 1D $t-J$ model with $t=1$ (main plot). Line shape at $q=\pi$ for various values of $J$ in the phase-separated state (inset).

(ii) The intensity of the central peak in $S_{z z}(\pi / 2, \omega)$ weakens gradually. This is illustrated in the main diagram of Fig. 2. The peak turns shallow and then vanishes altogether. This property of our data reflects the gradual weakening and ultimate disappearance of the power-law divergence (6), given the (approximately known) $J / t$ dependence of the infrared exponent.

As the coupling strength increases past the value $J / t \simeq 1$, the spin modes which dominate $S_{z z}(q, \omega)$ in the first regime of the Luttinger liquid phase broaden rapidly and lose their distinctiveness. There is a crossover region between the first and second regimes, which roughly comprises the coupling range $1 \lesssim J / t \lesssim 2$. Over that range, the spectral weight in $S_{z z}(q, \omega)$ is distributed over a broad structure with rapidly shifting peaks.

At the end of the crossover region, a new type of spin mode with an entirely different kind of dispersion has gained prominence in $S_{z z}(q, \omega)$, and it stays dominant throughout the remainder of the Luttinger liquid phase. This is illustrated in Fig. 3 by a plot of $S_{z z}(q, \omega)$ for a $J / t$ value near the onset of phase separation. The representation is the same as in Fig. 1 except for the different frequency scale.

The dispersion of these new spin modes, as shown by the full circles, does no longer have a soft mode at $q=\pi / 2$. At $J / t \sim 2.0$ the dispersion has a $|\sin (q / 2)|$-like shape, i.e., a smooth maximum at $q=\pi$ and a tendency to approach zero linearly as $q \rightarrow 0$. As $J / t$ increases toward the transition point, the peak positions in $S_{z z}(q, \omega)$ gradually shift to lower values of $\omega / J$ (with $t$ held fixed). The shift proceeds more rapidly at $q$ near $\pi$ than at smaller $q$, which has the consequence that the maximum in the dynamically relevant spin dispersion starts to move away from $q=\pi$ toward $q=\pi / 2$. 


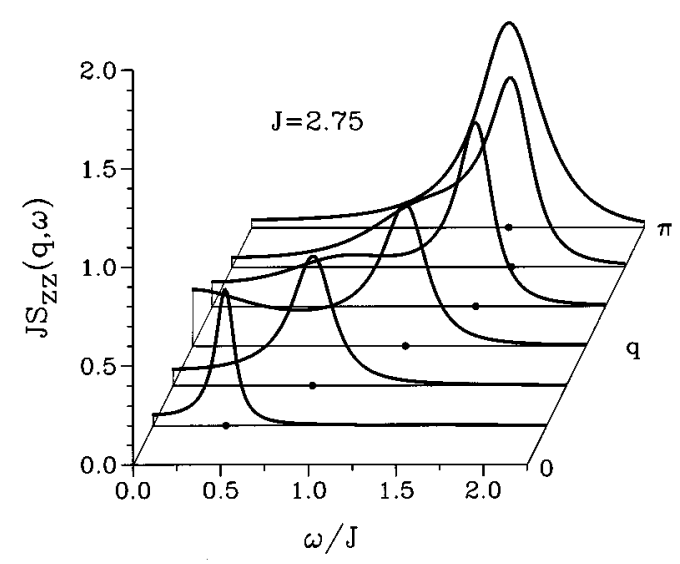

FIG. 3. Spin dynamic structure factor $S_{z z}(q, \omega)$ at $T=0$ for $q=2 \pi l / 12$, $l=0, \ldots, 6$ of the $1 \mathrm{D} t-J$ model with $t=1$ and $J=2.75$ in the Luttinger liquid phase near the onset of phase separation.

This trend from a $|\sin (q / 2)|$-like toward a $|\sin q|$-like dispersion persists in the phase-separated state $(J / t>3.2)$.

The evolution of the spectral-weight distribution in $S_{z z}(\pi, \omega)$ from a well-defined mode at $\omega / J \simeq 1.4$ near the onset of phase separation toward a soft mode in the strongly phase-separated state is shown in the inset to Fig. 2. By contrast, the spectral weight in $S_{z z}(\pi / 2, \omega)$, which has been slowly shifting toward lower values of $\omega / J$ in the second regime of the Luttinger liquid phase, now starts to move back out to higher frequencies in the phase-separated state (not shown here). The single-peak structure grows taller, the linewidth shrinks somewhat, and the peak position settles at $\omega / J \simeq 1.57$.

All these properties and trends reflect or foreshadow the much better understood $T=0$ dynamical properties of the 1D $s=1 / 2$ Heisenberg antiferromagnet - the end product of the
$t-J$ model in the completely phase-separated state. The spin dynamic structure factor $S_{z z}(q, \omega)$ under these circumstances is known to be dominated by a continuum of two-spinon excitations with a lower boundary $\epsilon_{L}(q)=(\pi / 2) J|\sin q|$, where the spectral-weight distribution has a divergent singularity, and an upper boundary $\epsilon_{U}(q)=\pi J|\sin (q / 2)|$, where the spectral-weight distribution becomes very small. ${ }^{7,8,13}$

This work was supported by the U.S. National Science Foundation, Grant No. DMR-93-12252. Computations were carried out on supercomputers at the National Center for Supercomputing Applications, University of Illinois at UrbanaChampaign.

${ }^{1}$ F. C. Zhang and T. M. Rice, Phys. Rev. B 37, 3759 (1988); C. Gros, R. Joynt, and T. M. Rice, Phys. Rev. B 36, 381 (1987).

${ }^{2}$ At $J / t=0$ it is more likely that the site adjacent to an electron is occupied by a hole than by another electron.

${ }^{3}$ M. Ogata, M. U. Luchini, S. Sorella, and F. F. Assaad, Phys. Rev. Lett. 66, 2388 (1991).

${ }^{4}$ F. F. Assaad and D. Würtz, Phys. Rev. B 44, 2681 (1991).

${ }^{5}$ C. S. Hellberg and E. J. Mele, Phys. Rev. Lett. 67, 2080 (1991).

${ }^{6}$ R. Haydock, Solid State Physics 35, 215 (1980); M. H. Lee, Phys. Rev. B 26, 2547 (1982); E. R. Gagliano and C. A. Balseiro, Phys. Rev. B 38, 11766 (1988).

${ }^{7}$ V. S. Viswanath and G. Müller, Recursion Method_Application to ManyBody Dynamics, Lecture Notes in Physics, Vol. m 23 (Springer-Verlag, New York, 1994).

${ }^{8}$ V. S. Viswanath, S. Zhang, J. Stolze, and G. Müller, Phys. Rev. B 49, 9702 (1994).

${ }^{9}$ V. S. Viswanath, S. Zhang, G. Müller, and J. Stolze, Phys. Rev. B 51, 368 (1995).

${ }^{10}$ J. Deisz, K.-H. Luk, M. Jarrell, and D. L. Cox, Phys. Rev. B 46, 3410 (1992); T. Tohyama, P. Horsch, and S. Maekawa, Phys. Rev. Lett. 74, 980 (1995).

${ }^{11}$ N. Kawakami and S.-K. Yang, Phys. Rev. Lett. 65, 2309 (1990).

${ }^{12}$ A. Luther and I. Peschel, Phys. Rev. B 12, 3908 (1975); H. C. Fogedby, J. Phys. C 11, 4767 (1978).

${ }^{13}$ G. Müller, H. Thomas, H. Beck, and J. C. Bonner, Phys. Rev. B 24, 1429 (1981). 